\title{
La francophonie au Congo-Kinshasa : pratiques ordinaires et littéraires (1945-1970)
}

Luc Collès

\section{(2) OpenEdition \\ 1 Journals}

Édition électronique

URL : https://journals.openedition.org/dhfles/127

DOI : $10.4000 /$ dhfles. 127

ISSN : 2221-4038

Éditeur

Société Internationale pour l'Histoire du Français Langue Étrangère ou Seconde

Édition imprimée

Date de publication : 1 janvier 2008

Pagination : 199-210

ISSN : 0992-7654

Référence électronique

Luc Collès, «La francophonie au Congo-Kinshasa : pratiques ordinaires et littéraires (1945-1970) »,

Documents pour l'histoire du français langue étrangère ou seconde [En ligne], 40/41 | 2008, mis en ligne le 17 décembre 2010, consulté le 27 mai 2021. URL : http://journals.openedition.org/dhfles/127 ; DOI :

https://doi.org/10.4000/dhfles.127

Ce document a été généré automatiquement le 27 mai 2021.

(C) SIHFLES 


\title{
La francophonie au Congo- Kinshasa : pratiques ordinaires et littéraires (1945-1970)
}

\author{
Luc Collès
}

1 La République démocratique du Congo (RDC) - devenue le Zaïre entre 1970 et 1997 et appelée aussi le Congo-Kinshasa pour le différencier du Congo-Brazzaville (ou République du Congo) - compte actuellement 4 millions de francophones (sur 42 millions d'habitants), soit à peine $10 \%$ de la population. Pendant trois-quarts de siècle, jusqu'en 1960, ce pays, comme le Rwanda et le Burundi, a vécu sous influence belge. C'est la Belgique qui y a introduit le français.

\section{Histoire du français}

2 Depuis 1877, la langue française a été de facto la langue officielle, administrative et judiciaire du Congo. Cependant, lorsque l'État Indépendant du Congo devint en 1907 une colonie belge, le problème linguistique commença à se poser. Des débats houleux opposèrent, au sein du Parlement belge, les partisans de la langue française et ceux qui voulaient garantir les droits des Flamands en exigeant que soit proclamée une égalité linguistique dans la Colonie entre les deux langues nationales (Band1956: 424-450). Mais, bien qu'il fût décidé que tous les décrets seraient alors rédigés en français et en néerlandais, il s'est installé une situation de fait faisant du français la seule langue officielle du Congo. Si cette exclusion (de facto mais non de jure) du néerlandais tant de la vie officielle que de l'enseignement a pu être tolérée, c'est que pratiquement tous les Belges flamands d'un niveau social plus ou moins élevé connaissaient fort bien le français.

3 Selon Max Pierre (1997: 318-321), on peut distinguer dans l'histoire du français au Congo-Kinshasa cinq périodes bien définies. Ces périodes sont celles qui scandent l'évolution de l'enseignement. C'est par l'apprentissage scolaire principalement que le français est acquis par la plupart de ses locuteurs congolais. Cependant, l'apprentissage 
du français s'est réalisé aussi par les contacts sociaux noués entre Européens et Africains, notamment dans les rapports professionnels (ouvriers, domestiques) et dans les aspects plus personnels de la vie commune : activités culturelles, œuvres sociales. Mais ce niveau d'apprentissage ne débouchait que sur un français limité.

La première période, qui va des débuts de la colonisation à 1948, est caractérisée par un mouvement d'alternance entre la thèse des européanistes et celle des indigénistes (Faïk 1988 : 12-16). D'une part, les européanistes sont partisans de l'imposition du français comme langue de communication, ce qui simplifie les choses dans un pays qui compte plus de deux cents langues. D'autre part, les indigénistes sont partisans des langues africaines, pour diverses raisons : les Flamands, pour ne pas favoriser uniquement le français ; d'autres pour assurer le sauvetage des valeurs authentiquement congolaises ; d'autres enfin dissimulaient mal leur désir de maintenir la population autochtone dans un état d'infériorité politique.

5 Les missionnaires, convaincus qu'une langue étrangère ne pouvait être le véhicule d'une évangélisation efficace, se sont les premiers intéressés aux parlers autochtones. Or, si l'on sait que presque tout le système scolaire était entre leurs mains, on comprendra que bien vite ils s'orienteront vers un enseignement en langues locales. La situation au Congo-Kinshasa était donc fort différente de celle des colonies françaises qui avaient opté pour l'assimilation. Sous le régime colonial français, en effet, l'enseignement était obligatoirement dispensé en français, dans toutes les matières et à tous les niveaux (Champion $1974: 260$ ).

6 Mais la multiplicité des langues constituant un handicap sérieux, on songea à s'orienter vers une des quatre langues à vocation nationale : le kikongo, le lingala, le cilubà et le swahili. Un arrêté royal du 10 juillet 1922 officialise ce principe de l'enseignement en langues autochtones. Une enquête (De Jonghe 1933: 516-517) révèle que, vers cette date, dans les petits séminaires, la langue indigène est utilisée comme véhicule de l'enseignement pendant les trois premières années du secondaire et n'est remplacée par le français qu'à partir de la quatrième. Par après s'opéra une certaine marche arrière et l'on finit par adopter une sorte de compromis entre «l'européanisme » des premiers temps et « l'indigénisme » qui suivit. Le programme de 1948 peut se rattacher à cette solution du juste milieu (Faïk 1988 : 15-16).

7 Les deux premières années de l'enseignement primaire se font en langue locale. Durant les trois années suivantes, l'enseignement se donne toujours en langues locales, mais l'enseignement du français y est introduit. Au cours d'une sixième année (facultative) préparatoire à la section moyenne, l'enseignement du français s'intensifie. Le programme de 1948 impose le français comme langue d'enseignement et comme première langue étudiée (sept à dix heures par semaine) dans l'enseignement secondaire, tout en maintenant l'étude d'une langue culturelle congolaise de grande expansion.

8 Durant la deuxième période, entre 1948 et 1960 (Pierre 1997: 320), on assiste à une accélération de la politique européaniste et à une intensification de l'usage du français. Les écoles moyennes passent de quatre à six ans et inscrivent à leur programme l'étude de la littérature française. Plusieurs périodiques rédigés en français s'adressent au public africain : La Croix du Congo, La Voix du Congolais, Actualités africaines à Kinshasa. C'est en 1955 que le ministre des colonies, Auguste Buisseret, introduit l'école laïque au Congo. Sa particularité est que l'enseignement y est entièrement dispensé en français alors que, dans l'enseignement confessionnel, seul existant au Congo de l'époque et 
monopole des missions catholiques et protestantes, l'enseignement primaire et normal est, comme nous l'avons vu, largement dispensé en langues vernaculaires. L'école nouvelle remporta un vif succès du fait, sans doute, que c'était la première fois que l'alternative laïque était offerte aux Africains. C'est aussi à cette époque qu'est créée l'université Lovanium à l'initiative de l'université de Louvain (Belgique) et à l'opposé des colonies voisines qui envoyaient les Africains poursuivre leurs études dans les universités européennes.

9 La troisième période commence en 1960, avec l'indépendance du pays. La bourgeoisie intellectuelle - enseignants et anciens séminaristes - accède au pouvoir et fait du français la langue officielle du pays. Dès 1962, une ordonnance présidentielle écarte les langues congolaises de l'école. Le français devient l'unique langue de l'enseignement du secteur primaire. Les nouveaux dirigeants politiques voulaient en effet conjurer le tribalisme et le pluralisme linguistique en favorisant l'emploi d'une «langue unificatrice». De plus, la forte centralisation administrative rendait nécessaire le recours à une langue considérée comme «neutre " parce que « non congolaise ». Enfin, la valorisation sociale du français, jadis refusée aux « indigènes ", exerça une profonde attraction chez les Congolais francophiles qui désiraient s'approprier cette langue prestigieuse et génératrice de développement économique.

10 Mais plusieurs coups d'arrêt sont donnés à cette euphorie francophone (Pierre 1997 : 321). En 1963-1964, la révolution paysanne de Mulele dans le Kwilu s'oppose à tout ce qui est étranger et bourgeois, et donc francophone ou francophile. En 1967, le congrès de Luluabourg remet en cause le français dans l'enseignement fondamental et prône un retour aux langues africaines. Les élites veulent affirmer la vitalité des cultures négroafricaines, mais, d'après Charles Tschimanga (2001:288), l'affirmation de ces valeurs ne pouvait suffire pour faire contrepoids à un système mondial dominé par les valeurs de l'Occident.

11 Cependant, à partir de l'instauration de la dictature mobutienne vers 1970, la connaissance et la pratique du français n'ont plus été des critères suffisants pour accéder au travail et aux postes de responsabilité. D'autres facteurs sont intervenus : militantisme politique, appartenance ethnique, pratique de la langue du chef et, dès lors, l'image du français liée à la promotion sociale et à la prospérité économique a changé. La quatrième période coïncide avec la politique « d'authenticité », lancée par le Président Mobutu pour galvaniser le nationalisme des Congolais: la République démocratique du Congo devient le Zaïre en 1970, les prénoms étrangers, donc français pour la plupart, sont remplacés par des noms africains : diplômes, intitulés de compte en banque, enseignes de magasin vont changer. Bref, la prise du pouvoir politique par Mobutu s'est traduite également par une prise du pouvoir linguistique.

12 Cette idéologie du recours à l'authenticité entraîna apparemment la valorisation des langues nationales et remit en question le monopole du français. Le domaine de l'enseignement fut partiellement touché. Après avoir été exclues depuis 1962, les quatre langues nationales furent réintégrées officiellement dans l'enseignement primaire, mais elles restèrent confinées aux deux premières années du primaire. Pour que le recours à l'authenticité devienne un nouvel ordre linguistique, il aurait fallu qu'il dépasse les formes superficielles et limitées des termes africanisés et qu'il consacrât les langues nationales comme des facteurs de développement social et économique.

13 Par ailleurs, c'est suite à la visite du président Giscard d'Estaing, en 1976, que sera décidée la construction de la "Voix du Zaïre », puissant émetteur de radio-télévision, 
financé par la France, et instrument non équivoque de la volonté de garder le pays dans le giron de la francophonie. Malgré la zaïrisation linguistique, le français revint en force et fut enseigné à l'oral à raison d'une demi-heure par jour dans les deux premières années du primaire et fut introduit progressivement à l'écrit en troisième année pour devenir l'unique langue d'enseignement en cinquième année. Au secondaire, les langues nationales n'ont jamais été enseignées.

La cinquième période commence en 1985 et marque le déclin de l'authenticité. Nous l'évoquons rapidement car elle sort du cadre chronologique de notre propos. Durant un colloque consacré au thème "Linguistique et Société ", juristes, politiciens, généraux plaident pour les langues nationales. Mais, un mois après, une circulaire du ministère de l'Éducation enjoint de renforcer le français : cette circulaire fut comprise par les inspecteurs comme entraînant la suppression des langues africaines dont l'enseignement avait été péniblement mis en place.

Le français est devenu l'unique langue du parlement et du gouvernement, mais aussi celle de l'Administration, de l'école, de la grande presse, etc., au détriment des langues nationales. C'est ce qui fait dire à Mwatha Musanji Ngalasso (1986:6-27), un linguiste d'origine congolaise, que, de ce point de vue-là, la francophonie au Congo, dans ce qu'elle a aujourd'hui de plus conquérant, est bien une invention postcoloniale. Cette politique du français s'est poursuivie, essentiellement par décrets, jusque vers le milieu des années 70. De fait, les Belges n'ont jamais voulu imposer le français aux « indigènes » sous prétexte de ne pas les déraciner.

\section{La francophonie au Congo-Kinshasa (de 1945 à 1960)}

Sur le terrain, la plupart des Congolais pratiquent donc une diglossie déséquilibrée, sinon une triglossie lorsqu'ils savent le français (Calvet 1998 : 7-38). Ainsi, ils emploient leur langue maternelle (ou ethnique) dans les relations familiales ou interethniques, mais la langue véhiculaire régionale (kikongo, lingala, swahili et cilubà) dans la vie urbaine en général : commerce, administration locale, éducation (premier cycle du primaire). Pour ceux qui savent le français (triglossie), employer cette langue donne automatiquement accès à toutes les sphères du pouvoir et de la connaissance. Le français est encore, dans les faits, la langue de l'État et du droit.

En dehors des secteurs officiel, scolaire et scientifique, c'est surtout dans les mass media que le français occupe une place privilégiée (Faïk 1988: 23). Le Congo-Kinshasa est membre de l'Union internationale des journalistes et de la presse de langue française. Les journaux sont presque exclusivement rédigés en français. Mais la situation est sensiblement différente à la radio et à la télévision où le français semble n'occuper que $60 \%$ du temps de parole. Quant aux chansons, selon des estimations assez sûres, elles sont en lingala, la chanson française étant pratiquement exclue. En revanche, les films, tant ceux de la télévision que ceux projetés dans les cinémas, sont toujours en français. Au Congo-Kinshasa, le français est donc beaucoup plus lu et entendu qu'il n'est parlé et écrit.

18 C'est dans le domaine culturel et particulièrement littéraire que ce pays ressortit à la francophonie. Comme le propose la thématique de ce colloque, nous allons, pour le montrer, nous limiter à la période allant de 1945 à 1970. C'est Silvia Riva qui, dans sa 
Nouvelle histoire de la littérature du Congo-Kinshasa (2006) et Mukala Kadima-Nzuji, dans La Littérature zaïroise française (1984) qui ont été nos principales sources d'informations.

Vers 1945, avec près de dix ans de retard sur l'Afrique française, de nombreuses associations d'anciens élèves virent le jour dans les plus grands centres urbains de la colonie belge, et en particulier à Léopold-ville, la capitale (aujourd'hui, Kinshasa). L'Administration belge confia au gouverneur Jean-Paul Quix la tâche d'instituer un comité de contrôle des activités culturelles. Celui-ci prendra le nom de La Voix du Congolais, du nom de la revue que fonda Quix. On peut retenir, sur la production poétique de cette revue, le jugement exprimé par Mukala Kadima-Nzuji (1986: 90): " conformisme des thèmes et du style, [...] complaisance dans une prosodie surannée. " Mais la rédaction de la revue découvrira le mouvement surréaliste et la Négritude à partir de 1953 et consacrera une série de monographies à des écrivains francophones confirmés : René Maran, Aimé Césaire, Birago Diop, Gilbert Gatiant, Martial Sinda, premier poète noir de l'AEF, etc. Ce sont ces essais qui représentent la véritable contribution de la revue à l'histoire littéraire du Congo-Kinshasa. L'ouverture au monde africain et à celui des Caraïbes servit de stimulation aux jeunes écrivains.

En 1946, à Élisabethville, une association culturelle est fondée, l'Union Africaine des Arts et des Lettres (l'UAAL), visant à " promouvoir et diffuser les ouvrages et les œuvres artistiques des écrivains et artistes établis au Congo belge et au Ruanda-Urundi ». Cette association entretenait des rapports étroits avec le reste du monde africain francophone grâce à la collaboration d'organisations internationales (surtout l'Unesco) et d'instituts privés français. Cette société disposait également d'un organe de presse, Jeune Afrique, qui se proposait, « partant du Congo, de construire à l'échelle de l'Afrique et de développer les qualités culturelles des peuples noirs en vue d'arriver à une meilleure compréhension de leurs aspirations spirituelles et à une interpénétration des continents par le rayonnement donné aux Arts et Lettres, source de pacifiques espérances ». La ligne éditoriale correspondait aux aspirations que nourrissait à ce moment le mouvement de la Négritude. Il n'est donc pas surprenant que le numéro d'octobre 1949 contienne une poésie de Léopold Sédar Senghor (" Teddungal ») ou que l'un des plus grands personnages de la Négritude congolaise, Antoine Roger Bolamba (auteur de Esanzo. Chants pour mon pays), ait trouvé en 1953 un grand espace dans les pages de Jeune Afrique pour exposer son esthétique.

21 Née à Leverville en 1943, la Bibliothèque de l'Étoile publiait des collections diverses dont l'une, "l'Étoile", se consacrait autant à la littérature et aux problèmes linguistiques qu'aux discussions scientifiques, historiques et sociales. On y trouvait des extraits et des traductions des classiques de la littérature française et mondiale (la Chanson de Roland, le Roman de Renart, les fables de La Fontaine, lî̂le au trésor de Stevenson), mais aussi des textes africains (par exemple des contes). La Bibliothèque de l'Étoile, au sein de ses collections, publiait également des romans dont victoire de l'amour (1954) de Dieudonné Mutombo et le Mystère de l'enfant disparu (1962) de Tomothée Malembe.

22 Mais les meilleurs résultats artistiques seront atteints lors de nombreux concours littéraires organisés au Congo belge entre 1946 et 1956. Ces concours consistaient, selon Mukala Kadima-Nzuji, en une réponse à deux types d'exigence $(1986: 199)$ : la première concernait le désir de l'Administration coloniale de favoriser au Congo l'envol de la littérature africaine dont la dignité était déjà reconnue en France (en 1947, André Gide, dans sa préface du premier numéro de la revue Présence africaine, conseillait d'être à 
l'écoute de ce que le peuple africain savait exprimer). La seconde résidait dans la volonté de montrer, de par les discours des ouvrages primés, les progrès effectués par le système colonial du Congo. C'est ainsi qu'en 1948, un concours lancé à l'occasion de la première Foire coloniale de Bruxelles, récompensa une œuvre qui fut considérée comme « le point de départ de la fiction romanesque au Zaïre» (Kadima-Nzuji 1986: 189) : Ngando de Paul Lomami Tchibamba. Léopold Sédar Senghor (à l'époque député) demanda une copie des manuscrits admis en finale affirmant sa conviction «que cela intéresserait par ailleurs Gide et le comité de patronage de Présence africaine.»

Lomami Tchibamba s'était déjà distingué en 1945 quand, dans La Voix du Congolais, il écrivit un article très polémique intitulé «Quelle sera notre place dans le monde de demain?» dans lequel il s'interrogeait sur les limites des libertés concédées aux "évolués». Il y dénonce l'Administration coloniale pour avoir imposé trop de restrictions et des langages brutaux. Une fois installé à Brazzaville pour le compte de Liaison (la «revue pour intellectuels » d'Afrique Équatoriale Française, qu'il dirigea entre 1950 et 1957), il écrit des articles encore plus enflammés en faveur de "l'émancipation du Noir ». Cette revue se présente comme une véritable « défense et illustration » d'une civilisation noire, en l'occurrence congolaise, tout à fait comparable à ce qu'entreprirent, dans le Paris des années trente, des écrivains prestigieux comme Césaire et Senghor. Le roman Ngando, et d'une certaine manière les autres textes de Lomami Tchibamba, se veut une réponse à l'ethocentrisme européen et en même temps une défense du patrimoine africain.

\section{Les années 1960}

L'obtention de l'indépendance a constitué pour le Congo, comme pour les autres pays africains, l'événement marquant d'une certaine époque qui, en tant que telle, représente une ligne de démarcation par rapport à l'époque précédente. La littérature des lendemains de 1960 présente toutefois un profil si diversifié qu'il rend une périodisation univoque difficile à établir (Riva 2006: 95). Bertin Makolo Muswaswa a opté pour une chronologie politique et propose de diviser la période sur laquelle court l'histoire littéraire du Congo-Kinshasa en trois phases : la phase coloniale (1908-1960), la phase de la Première République (1960-1965) et celle de la Seconde République (1965-1990). Mukala Kadima-Nzuji (1984) ne distingue, lui, que deux ères dans la production littéraire congolaise : de 1945 à 1965 (de La Voix du Congolais à la publication du roman Sans rancune de Thomas Kanza) puis la période suivante (arrêtée à 1990) qui court de la mise en place par les pouvoirs publics des Éditions Belles-Lettres, à l'éclosion de la presse liées aux exigences de démocratisation du Zaïre.

Les premières œuvres publiées après la libération se ressentent encore lourdement de l'héritage colonial, tant dans les choix narratifs (romans réalistes articulés autour d'un seul héros) que dans les thèmes (littérature de témoignage). Quelques-unes des compagnies de théâtre parmi les plus importantes du Congo indépendant sont fondées à Kinshasa à cette époque. La poésie fut aussi particulièrement féconde et l'art de l'essai (politique, philosophique, littéraire, social, religieux) s'affirme jusqu'à constituer l'une des principales contributions du Congo à la pensée africaine et mondiale.

Dès 1955, Bolamba exprime au niveau littéraire une rupture par rapport au ton complaisant envers l'autorité coloniale et révèle le mal-être et la colère de son peuple dans Esanzo. Chants pour mon pays. La production poétique des années 1960 oscillera 
constamment entre un courant ouvertement « révolutionnaire » et militant et un autre intimiste et hermétique dans lequel le " je » a le dessus. D'un côté, donc, Marche, pays des espoirs (1967) d'Étienne Tshinday Lukumbi et Réveil dans un nid de flammes (1969) de Matala Mukadi Tshiakatumba; de l'autre, Murmures (1968) et Kasalà (1969) de Clémentine Faïk-Nzuji, Les ressacs (1969) de Dieudonné (Mukala) Kadima-Nzuji, et Déchirures (1971) de V. Y. Mudimbe.

D'après S. Riva, (2006: 103) trois facteurs sont responsables du nouvel essor des Lettres congolaises: la grande diffusion de l'instruction (qui atteint finalement un niveau universitaire), la naissance de cercles et de revues culturels dans les grandes universités et la création d'une structure éditoriale parrainée par l'État. Un cercle culturel baptisé "Pléiade du Congo" naît en 1964 sur le campus universitaire de Lovanium (Kinshasa). Celui-ci comptait parmi ses principaux animateurs la jeune poétesse Clémentine Nzuji (Faïk-Nzuji) qui, en 1969, remporte au festival de Dakar le premier prix au concours de poésie L. S. Senghor. Linguiste confirmée et spécialiste de la tradition orale, elle a publié des récits, des nouvelles, une chronique et de nombreux recueils poétiques. Quant à la production poétique de V. Y. Mudimbe, si elle date du tout début des années 1960, elle n'a été publiée qu'à partir de la décennie suivante. Déchirures, par exemple, date de 1971.

En 1969, les Éditions Congolia font paraitre l'anthologie intitulée Les écrivains congolais consacrée aux écrivains lauréats du "Concours littéraire Léopold Sédar Senghor ». L'influence de grands auteurs africains francophones se fait sentir chez plusieurs Congolais de l'époque. Matala Mukadi Tshioakatumba et Philippe Masegabio avouent leur dette envers les grands maitres de la Négritude (Césaire, Senghor, Léon Damas), mais ils se démarquent aussi de l'anthropologie du mouvement. Ainsi, selon Jean-Louis Joubert (1986 : 99), l'intention de Masegabio, dans Somme première, de retracer pas à pas (à travers la juxtaposition de poèmes-réflexions) le chemin emprunté par une pensée à la recherche d'une somme improbable est plus proche de la voie suivie par Samba Diallo que du flux d'images instinctif et spontané d'un Senghor ou d'un Damas. Diallo est le protagoniste du roman L'aventure ambiguë, publié à Paris chez Julliard en 1961 par le Sénégalais Cheikh Hamidou Kane, lequel a interprété, le premier, la déchirure spirituelle d'une génération élevée au milieu de deux cultures apparemment incompatibles.

29 Face à l'effervescence de la production de poésies, mais aussi de pièces de théâtre et d'essais entre 1960 et 1970, le roman congolais marque le pas de manière flagrante. Seules cinq œuvres ont paru à cette période. Mis à part Sans rancune de Thomas Kanza, ces romans pêchent par manque de profondeur psychologique, l'important étant de mettre en lumière le risque d'aliénation encouru par le colonisé. Ces œuvres préparent surtout, à leur insu, un terrain propice au développement de l'idéologie de l'authenticité. La fracture entre élites occidentalisées et masses, évidente bien avant l'indépendance, se change, au début des années 1970, en fracture entre élites et pouvoir. Cette incompréhension entre le pouvoir et une partie des intellectuels aboutira, sur le plan social, à une fuite des cerveaux et, au niveau littéraire, à une représentation de l'expérience de la déchirure, qui influencera l'ensemble de la décennie suivante.

30 En conclusion, on peut globalement affirmer que depuis l'époque coloniale jusqu'à la décennie 1970, le français a été, au Congo-Kinshasa, la langue dont la maîtrise donnait accès au monde du travail, à un emploi mieux rémunéré et à l'ascension sociale. Mais il 
n'a jamais atteint le statut de langue vernaculaire parmi le peuple. Le français reste la langue de l'élite scolarisée qui représente environ $10 \%$ de la population nationale. C'est surtout par sa littérature que la République démocratique du Congo appartient à la francophonie. En particulier, les œuvres de Senghor et Césaire influenceront les écrivains congolais. L'Union Africaine des Arts et des Lettres, par exemple, entretenait dès 1946 des liens avec le mouvement de la Négritude. Après l'indépendance, plusieurs écrivains ont aussi avoué leur dette envers ce mouvement. Mais la littérature du CongoKinshasa a aussi son originalité et ses spécificités : nous espérons l'avoir montré par les différentes orientations que nous avons tracées.

\section{BIBLIOGRAPHIE}

Band(1956), n 10-11, Antwerpen, p. 424-450.

CALVET, Louis-Jean (1998). «L'insécurité linguistique et les situations africaines », in Louis-Jean Calvet et Marie-Louise Moreau (éds), Une ou des normes? Insécurité linguistique et normes endogènes en Afrique francophone, Paris, Agence de la francophonie, p. 7-38.

CHAMPION, Jacques (1974). Les langues africaines et la francophonie, Paris-La Haye, Mouton.

DE JONGHE (1933). « Les langues communes au Congo belge (I) », in Congo II, Bruxelles, p. 509-523.

FAÏK Sully et al. (1988). La Francophonie au Zaïre, Lubumbashi, Impala, p. 5-40.

JOUBERT, Jean-Louis (1986). Critique de « Somme première », in Notre Librairie, nº 63, p. 99.

KADIMA-NZUJI, Mukala (1984). La littérature zaïroise de langue française, Paris, Karthala.

NGALASSO, Mwatha Musanji (1986). «État des langues de l'État du Zaïre », in La Politique africaine, $\mathrm{n}^{\circ}$ 23, Paris, Karthala, p. 6-27.

PIERRE, Max (1997). « Le français au Zaïre, au Rwanda et au Burundi », in D. Blampain et al., Le français en Belgique, p. 319-333.

RIVA, Silvia (2006). Nouvelle histoire de la littérature du Congo-Kinshasa, Paris, L'Harmattan. TSHIMANGA, Charles (2001). Jeunesse, formation et société au Congo/Kinshasa : 1890-1960, Paris, L'Harmattan (coll. Congo-Zaïre - Histoire \& Société).

\section{RÉSUMÉS}

Depuis l'époque coloniale jusqu'à la décennie 1970, le français a été, au Congo-Kinshasa, la langue dont la maîtrise assurait l'ascension sociale. Mais il n'a jamais atteint le statut de langue vernaculaire parmi le peuple. Le français reste la langue de l'élite scolarisée qui représente $10 \%$ de la population nationale.

C'est surtout par sa littérature que la République démocratique du Congo appartient à la francophonie. En particulier, les œuvres de Senghor et Césaire influenceront les écrivains congolais. L'Union Africaine des Arts et des Lettres, par exemple, entretenait dès 1946 des liens avec le mouvement de la Négritude. Après l'indépendance, plusieurs écrivains ont aussi avoué 
leur dette envers ce mouvement. Mais la littérature du Congo-Kinshasa a aussi son originalité et ses spécificités que nous serons amenés à préciser.

From colonial times until the seventies, French was, in Congo-Kinshasa, the language which assured social ascent. But it has never attained the status of a vernacular language among the people. French remains the language of the schoolgoing elite which represents $10 \%$ of the national population.It is especially by its literature that the democratic Republic of Congo belongs to the French-speaking world. Particularly, the writings of Senghor and Césaire will influence the Congolese writers. The African Union of Arts and Letters, for instance, maintained from 1946 links with the movement of Negritude. After independence, several writers also confessed their debt towards this movement. But the literature of Congo-Kinshasa also has its originality ans its peculiarities which we shall be led to specify.

INDEX

Keywords : French as a language of the social ascent, links with the negritude movement, specificities of the literature of Congo-Kinshasa

Mots-clés : français comme langue de l'ascension sociale, liens avec le mouvement de la négritude, spécificités de la littérature du Congo-Kinshasa

\section{AUTEUR}

\section{LUC COLLÈS}

UCL - CRIPEDIS, Louvain-la-Neuve, Belgique

luc.colles@uclouvain.be 